\title{
XVI
}

(Aus dem St. Louis Skin and Cancer Hospital.)

\section{Die Krebskrankheit in den Vereinigten Staaten von Nordamerika.}

\author{
Von \\ Dr. Guthrie McConnell. \\ (Mit einer Karte der Vereinigten Staaten.)
}

Die nachfolgende Studie über die Verbreitung des Krebses in Nordamerika basiert auf den Erhebungen des Statistischen Amtes der Vereinigten Staaten und auf den Ergebnissen der letzten Volkszählung im Jabre 1900. Unter der Bezeichnung "Krebs" sollen auch alle andern malignen Tumoren zusammengefasst werden.

Es galt zunächst, festzustellen, ob die in anderen Ländern beobachtete Zunahme der Krebskrankheit auch für die Vereinigten Staaten zutrifft. Es ist ja bekannt, dass die Annahme eines Anwachsens der Krankheit auf lebhaften Widerspruch gestossen ist. Die Gegner dieser Anschauung wenden ein, dass die Zunahme nur eine scheinbare sei. Sie behaupter, die gegenwärtigen statistischen Werte seien nur der Ausdruck einer Vervollkommnung der diagnostischen Methoden sowohl, wie der Registrierung der Fälle. Derartige Einwände mögen bis zu einem gewissen Grade sticlıhaltig sein, wenn man die heutigen Zahlen mit denen vor vierzig und fünfzig Jahren vergleicht. Unsere Methoden sind in jeder Beziehung genauer und mögen zum Teil für die Zunahme verantwortlich sein. Berücksichtigt man jedoch nur die Werte der letzten zehn Jahre, so können die Einwände nicht mehr in dem gleichen Masse geltend sein. Die Vervollkommnung der Diagnose hat innerhalb dieses Zeitranmes nicht mehr solche glänzende Fortschritte gemacht.

Des weiteren wurden mit der Verbesserung der Laboratoriumsmethoden viele Tumoren als gutartige erkannt, die bisher als bösartige Neubildungen entferut worden waren. Es wäre zu erwarten, dass eine exaktere Diagnose 
die Zahl der Krebsfälle demgemäss eher vermindern würde. Anstatt jeden Tumor in einer sterbenden Person als die Todesursache und somit als bösartig anzusehen, wird jetzt seine wahre Natur erkannt.

Zum Vergleich wurden in Tabelle 1 die Mortalitätsziffern für Lungenschwindsucht und Pneumonie zu der von Krebs hinzugefügt. Diese Tabelle gibt die Zahl der Todesfälle in den Vereinigten Staaten nach den Zählungen der Jahre 1900 und 1890 1. nach ibrer Frequenz geordnet und 2. im Verhältnis von je einer Ursache zu 100000 von allen Todesursachen.

$\mathrm{T} a \mathrm{~b}$ elle 1 .

\begin{tabular}{|c|c|c|c|c|c|}
\hline \multirow{2}{*}{ Todesursache } & \multirow{2}{*}{$*$} & \multicolumn{2}{|c|}{1900} & \multicolumn{2}{|c|}{1890} \\
\hline & & Zahl & Verhältnis & Zahl & Verhältnis \\
\hline Lungenschwindsucht . & . & 111,059 & 10,688 & 102,199 & 12,146 \\
\hline Pneumonie. . . & & 105,971 & 10,198 & 76,496 & 9,091 \\
\hline Krebs . . . & & 29,475 & 2,837 & 18,536 & 2,203 \\
\hline
\end{tabular}

Die amtliche Statistik, welche der vorstehenden Tabelle entnommen ist, zeigte gegen 1890 eine Zunahme der Mortalität an Pneumonie, Herzkrankheiten, Nierenkrankheiten, Typhus und Krebs und eine Abnahme bei Lungenschwindsucht und Darmlkatarrhen. In dieser Tabelle figuriert Krebs an sechster Stelle.

Bevor wir in weitere Einzelheiten gehen, scheint es angebracht, einige Erläuterungen über die Art und Weise zu geben, mit der diese amtlichen Erhebungen angestellt wurden. Das Statistische Amt in Washington hatte eine Art von Fragebogen als die Grundlage zuverlässiger statistischer Nachweise für das Volkszählungsjahr 1900 festgesetzt. Dieser Fragebogen, der eine grosse Reihe von sorgfältig ausgeführten Erhebungen voraussetzte, wurde an alle Staaten und grössere Städte der Union ausgeschickt, aber nur eine gewisse Anzahl von Staaten und Städten war in der Lage, den gestellten Anforderungen voll und ganz zu genügen. Diese Staaten und Städte bilden zusammen den sogenannten Zählbezirk. Die Staaten, die zum Zählbezirk gehören, sind Maine, Vermont, New Hampshire, Massachusetts, Rhode Island, Connecticut and Michigan mit allen ibren Städten und ländlichen Bezirken. Die übrigen Staaten der Union komnten aus dem oben angeführten Grunde nicht in Betracht gezogen werden. Dagegen waren innerhalb dieser letztgenannten Staaten insgesamt fünfzig Städte, welche den amtlichen Anforderungen gerecht werden konnten und daber unter der Bezeichnung „Zählstädte in anderen Staaten" in den Zählbezirk und die amtliche Statistik aufgenommen wurden. $\mathrm{Zu}$ dieser letzten Klasse gehören fast alle grossen Städte, z. B. Philadelphia, St. Louis, Baltimore, San Francisco, Milwaukee, St. Paul, New Orleans. 
Für die Zähljahre 1900 und 1890 verhielt sich die Anzahl der Todesfälle an gewissen Krankheiten und die prozentuale Rate einer Krankheit, auf je 100000 Einwohner berechnet, wie folgt: 1890 starben an Krebs im ganzen 9410 Personen, d. h. 47,9 pCt. Zu dieser Zeit kam Krebs an dreizehnter Stelle in der Liste der Todesursachen. 1900 gab es 17296 Todesfälle mit einem Verhältnis von 60,0 an siebenter Stelle auf der Liste. Für die entsprechenden Jahre betrugen die Werte für Lungenschwindsucht 1890 . 48236 Todesfälle mit einem Verhältnis von 245,4; 190054898 Todesfälle mit einem Verhältnis von 190,5. Von der ersten Stelle sank sie zur zweiten auf der Liste. Für Pneumonie waren 189036752 Todesfälle bzw. 186,9 pCt., 190055296 Todesfälle bzw. 191,9 pCt. notiert, wodurch die Lungenschwindsucht aus der ersten Stelle verdrängt wurde. Die Tabellen, aus denen diese Ziffern entnommen wurden, zeigen eine Vermehrung in der relativen Sterblichkeit an Krankheiten, die am bäufigsten bei Erwachsenen und im vorgerückten Alter vorkommen und eine entschiedene Abnahme der Todesursachen in der Kindheit und der frühen Jugend. In Tabelle 2 ist die Mortalität in den Zäblbezirken mit den entsprechenden Unterabteilungen auf 100000 der Bevölkerung angegeben, und zwar von 1900 . im Vergleich mit 1890.

Tabelle 2.

\begin{tabular}{|c|c|c|c|c|c|c|}
\hline & \multicolumn{2}{|c|}{ Pneumonie } & \multicolumn{2}{|c|}{ Lungenschwindsucht } & \multicolumn{2}{|c|}{ Krebs } \\
\hline & 1900 & 1890 & 1900 & 1890 & 1.900 & 1890 \\
\hline Zählungsbericht & 191,9 & 186,9 & 190,5 & 245,4 & 60,0 & 47,9 \\
\hline$" \quad$ Städte 1 ) & 210,5 & 201,4 & 208,2 & 265,6 & 58,3 & 47,1 \\
\hline " Staaten im ganzen & 193,3 & 197,3 & 179,4 & 249,0 & 62,1 & 50,9 \\
\hline Städtische Bevölkerung. . . & 233,1 & 234,1 & 249,0 & 208,7 & 59,8 & 51,3 \\
\hline Landbevölkerung $\cdot \cdot$. . & 135,9 & 141,1 & 137,1 & 181,0 & 65,3 & 50,3 \\
\hline $\begin{array}{l}\text { Zählung in Städten anderer } \\
\text { Staaten } . . . . . .\end{array}$ & 189,9 & 171,1 & 207,7 & 239,9 & 57,0 & 43,2 \\
\hline
\end{tabular}

Aus dieser Tabelie ist ersichtlich, dass die Abnahme in der Mortalitätsziffer auf 100000 der Bevölkerung für Lungensehwindsucht in den zehn Jahren 54,9 betrug. In derselben Zeit nahm die Sterblichkeitsziffer an Krebs um 12,1 und an Pneumonie um 5,0 auf $100000 \mathrm{zu}$, wie in Tabelle 3 . gezeigt wird.

1) Es ist $z u$ beachten, dass in obiger und anderen Tabellen die Bezeichnung „Zählstädte" sich zunächst auf sämtliche Städte innerhalb des 'Zählbezirks be-zieht. Weiterhin werden diese Zählstädte eingeteilt in solche innerhalb der Zählstaaten und solche in anderen, nịcht dem Zählbezirk angehörigen Staaten. 
Tabelle 3 .

\begin{tabular}{|c|c|c|c|c|c|c|}
\hline$\theta$ & $\operatorname{im}_{\text {ganzen }}$ & Städte & 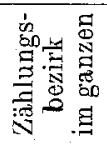 & 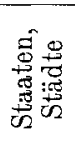 & 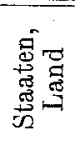 & 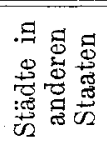 \\
\hline $\begin{array}{l}\text { Lungenschwindsucht, Abnahme } \\
\text { Pneumonie, Zunahme. } \\
\text { Krebs, Zunahme . } . .\end{array}$ & $\begin{array}{r}54,9 \\
5,0 \\
12,1\end{array}$ & $\begin{array}{r}57,4 \\
9,1 \\
11,2\end{array}$ & $\begin{array}{l}69,6 \\
\left.4,0^{1}\right) \\
11,2\end{array}$ & $\begin{array}{r}84,8 \\
1,0 \\
8,5\end{array}$ & $\begin{array}{r}43,9 \\
5,2 \\
15,0\end{array}$ & $\begin{array}{l}32,2 \\
18,8 \\
13,8\end{array}$ \\
\hline
\end{tabular}

Entsprechend den Werten für die Zunahme kommt Krebs an fünfter Stelle, während Nierenkrankheiten, Influenza, Apoplexie und Herzkrankheiten vorausgehen. Die folgenden Tabellen und Schlussfolgerungen behandeln eingebender die sozialen und ökonomischen Verhältnisse beim Krebs.

Zuerst sollen die Todesfälle an Krebs und Tumoren berücksichtigt werden, da es unmöglich ist, diese bei der üblichen Berichterstattung auseinander zu halten. Andere Momente sollen im Besonderen bei den Zahlen für die einzelnen Zählbezirke Berücksichtigung finden.

In Tabelle 4 werden die Todesfälle an Krebs für das Zähljahr 1900 gegeben.

Tabelle 4 .

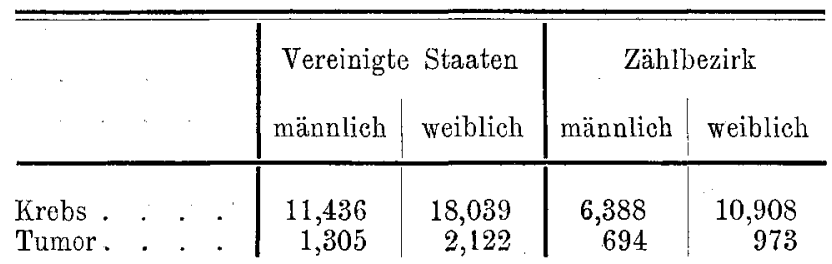

Die Gesamtsumme betrug 32902 , von denen 12741 männlich und 20161 weiblich waren. Das Verhältnis dieser Todesfälle zu 1000 Todesfällen an bekannten Ursachen betrug 32,9. Im Jahre 1890 betrug das entsprechende Verhältnis 25,4 .

Im Zählbezirk betrug die Zahl der Todesfälle an Krebs und Tumoren 18963, und zwar 7082 männlich und 11881 weiblich mit einem Verhältnis zu je 1000 Todesfällen von 37,4. Auf 100000 der Bevölkerung betrug die Sterblichkeitsziffer 65,8. Im Jahre 1890 betrug das Verhältnis 53,1 .

In den Zähljahren 1900 und 1890 zeigt die Sterblichkeitsziffer für Krebs im Zählbezirk mit seinen Unterabteilungen, dass die Sterblichkeit an diesen beiden Krankbeiten am höchsten in den ländlichen Distrikten

1) Abnahme.

Zeitsehrift für Krebsforschung. 7. Bd. 1. Heft. 
der Zählstaaten war (1900 71,4 anf 100000; 56,0 im Jahre 1890) und am niedrigsten in den Städten der ausserhalb des Zählbezirkes liegenden Staaten. Die Mortalität war höher unter den Weissen (66,7 im Jahre 1900; 53,9 im Jahre 1890) als bei den Farbigen (47,7 im Jahre 1900; 36,7 im Jahre 1890). Sie war viel höher bei den Nachkommen eingeborener Weisser (66,9 im Jahre $1900 ; 58,6$ im Jahre 1890) als bei denen von fremden . Eltern (25,7 im Jahre $1900 ; 17,9$ im Jahre 1890) und am höchsten bei den eingewanderten Weissen (124,6 im Jahre 1900; 93,3 im Jahre 1890.

Die verhältnismässig höhere Sterblichkeitsziffer für Frauen (weisse 83,2 für 1900; 69 für 1890; farbige 66,2 für $1900 ; 53,6$ für 1890) als für Männer (weisse 50,1 für 1900, 38,8 für 1890; farbige 28,6 für 1900, 19,2 für 1890) liegt an der grösseren Neigung dieser Krankheit, die Brust und die Zeugungsorgane der Frau zu ergreifen. Im Vergleich mit dem Jabre 1890 war eine entschiedene Zunahme der Sterblichkeitsziffer für Krebs und Tumor zu verzeichnen, und zwar vou $15 \mathrm{pCt}$. in den Städten der Zählbezirke bis zu 27 pCt. in den ländlichen Bezirken.

Es sollen nunmehr die Todesfalle an Krebs allein berücksichtigt werden, und zwar aus dem Zählbezirk und seinen Unterabteilungen. (Genauere Angaben über den Begriff „Zählbezirk" sind bereits oben erfolgt.)

Die Zählstaaten ordnen sich nun folgendermassen nach der Mortalitätsziffer für Krebs berechnet im Verhältnis zu je 100000 der Bevölkerung:

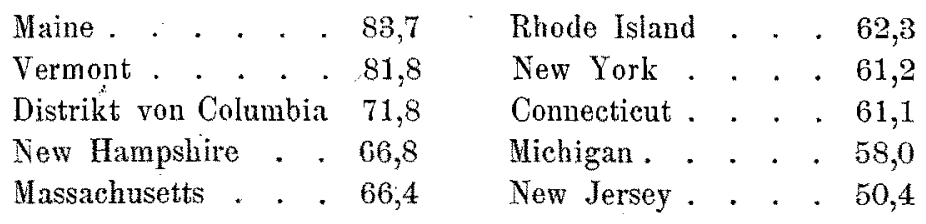

Die durchschnittliche Mortalitätsziffer für diese Staaten war nach Distrikten und Geschlecht berechnet:

\begin{tabular}{|c|c|c|c|c|}
\hline & & & Männer & Frauen \\
\hline 8 ta & . & 59,8 & 41,7 & \\
\hline an & . & 65,3 & 47.7 & 83,7 \\
\hline
\end{tabular}

Die höchste Sterblichkeit $(113,3)$ fand sich unter den Frauen in den ländlichen Bezirken von New Hampshire, die niedrigste $(31,5)$ unter den Mannern in den Städten von New Hampshire. Die Gesamtsterblichkeit an Krebs allein in den Vereinigten Staaten im Zahljahre 1900 betrug 29475 , und zwar 11466 Männer und 18039 Fraven, das Verhältnis der Todesfälle an dieser Krankheit zn je 1000 Todesfallen betrug 29,5. Die entsprechende Ziffer im Jahre 1890 betrug 22,5. Im Zählbezirk gab es 17296 Todesfälle an Krebs, davon 6388 Männer und 10908 Frauen; d. h. unter je 1000 Todesfälen war in $34,1 \mathrm{Krebs}$ die Ursache 
und von je 100000 Einwohnern starben 60 an Krebs. Im Jahre 1890 betrug letztere Mortalitätsziffer 47,9. Das Verbältnis von 34,1 setzt sich zusammen aus 12,58 oder 36,9 pCt. für Männer und 21,51 oder 63,1 pCt. für Frauen. Von den 17296 Todesfällen betrafen 16758 Weisse und 538 Farbige. In England und Wales betrug die Mortalität an Krebs im Jahre 1899 82,9 (Uänner 67,2; Frauen 97,7). Die Zunahme der Mortalitä.t in England von 1890 bis 1899 (15,3 pro 100000) war grösser, als die Zunahme im Zählbezirke der Vereinigten Staaten von 1890 bis 1900 (12,1). In Tabelle 5 wird die Mortalität an Krebs während des Zähljahres 1900 für den Zahlbezirk mit seinen Unterabteilungen gegeben, und zwar pro 100000 der weissen Bevölkerung nach Geburtsorten der Mütter geordnet.

Tabelle 5 .

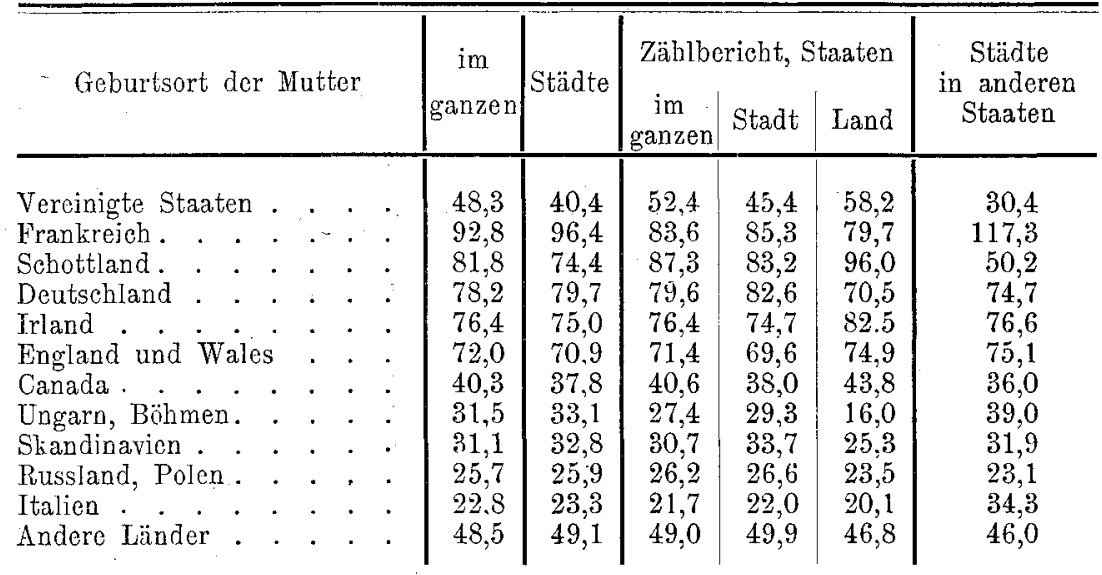

Diese Tabelle zeigt, dass die Mortalität an Krebs bei denen am höchsten war, deren Mutter in Frankreich geboren waren, 92,8; in Schottland 81,8 ; und in Deutschland 78,2 ; am niedrigsten bei denen, deren Mütter in Skandinavien geboren waren, 31,3; in Russland und Polen 25,7 und in Italien 22,8.

\section{Der Krebs in Städten.}

Die Zunabme der Mortalität in den Städten und auf dem Lande hat nicht gleichen Schritt gehalten, insofern nämlich, als in den ländlichen Bezirken die Zunahme gegen 1890 entschieden schneller stattfand, als dies in den Städten der Fall war. Im Jahre 1902 standen die folgenden fünf Städte an der Spitze hinsichtlich der Zahl der Todesfälle an Krebs auf je 1000 Todesfälle berechnet: 


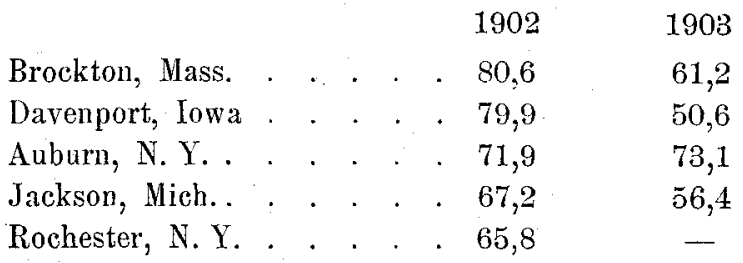

Im Jahre 1903 stauden die folgenden fünf Städte an der Spitze:

\begin{tabular}{|c|c|c|c|c|}
\hline & & & 1903 & 1902 \\
\hline Saginaw, Mich. & - & & 73,2 & 65,5 \\
\hline Malden, Mass. . & & & 73,1 & 57,0 \\
\hline Auburn, N. Y. . & - & & 73,1 & 71,9 \\
\hline Quincy, Mass. . & . & & 69,4 & 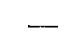 \\
\hline Grand Rapids, Mich. & . & & 69,2 & 59, \\
\hline
\end{tabular}

Hinsichtlich der Grösse der Städte betrug die Mortalität in den Jahren 1902 und 1903 auf 1000 Todesfälle in den zehn grössten Städten:

\begin{tabular}{|c|c|c|c|c|c|c|c|}
\hline & & & & & & 1903 & 1902 \\
\hline New York. & . & . & . & & · & . $\quad 38,7$ & 35,9 \\
\hline Chicago. & . & . & . & & . & . $\quad 41,9$ & 44,4 \\
\hline Philadelphia & . & . & . & 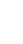 & . & . $\quad 38,2$ & 38,2 \\
\hline St. Louis & . & . & . & & . & . 40,3 & 31,8 \\
\hline Boston & . & . & . & & . & . $\quad 50,9$ & 45,7 \\
\hline Baltimore & . & . & . & & . & 38,3 & 39,8 \\
\hline Cleveland & . & . & . & . & . & . $\quad 37,4$ & 34,3 \\
\hline Buffalo . & . & . & . & & . & . 47,0 & 45,3 \\
\hline San Francisco & . & . & . & & . & . 58,7 & 59,4 \\
\hline Cincinnati . & . & . & . & & . & . 44,5 & 43,2 \\
\hline
\end{tabular}

Es scheint daber, dass die Sterblichkeit an Krebs geringere Nittelwerte in den grösseren Städten zeigt, als in den kleineren.

\section{Die Mortalität in verschiedenen Altersstnfen.}

Nach Abschnitten von vier Jahren geordnet, zeigt sich eine allmähliche Zunahme der Mortalität bis zu 25-29 Jabren. Dann folgt ein rapider Anstieg bis zu 55 Jahren, worauf die Zahl bis zu 70 Jahren fast stationär bleibt, von welchem Zeitpunkt an die Mortalität rapide zu sinken beginnt. (Siehe Tabelle 6).

Diese Zahlen Iebren, dass die Sterblichkeit an Krebs für Frauen in einer früheren Periode als bei Männern eine rapide Zunahme zeigt, ferner dass die Sterblichkeit bei Frauen der bei Männern um eine vierjährige Pcriode voraus ist, bis die Periode 55-59 erreicht ist, wo die Zahlen 
Tabelle 6.

Tod in den einzelnen Altersstufen auf 1000 Todesfälle.

\begin{tabular}{r|r|r|r|r}
\hline \multirow{2}{*}{ Alter } & \multicolumn{2}{|c|}{1900} & \multicolumn{2}{|c}{1890} \\
& Männer & Frauen & Männer & Frauen \\
& & & & \\
$20-24$ & 7,5 & 4,3 & 6,5 & 7,0 \\
$\mathbf{2 5 - 2 9}$ & 10,5 & 13,3 & 15,7 & 16,3 \\
$30-34$ & 19,9 & 30,8 & 23,7 & 39,0 \\
$35-39$ & 34,5 & 60,4 & 43,8 & 59,3 \\
$40-44$ & 58,0 & 89,3 & 54,2 & 95,2 \\
$45-49$ & 84,1 & 115,6 & 86,3 & 115,7 \\
$50-54$ & 117,5 & 129,8 & 111,6 & 133,5 \\
$55-59$ & 131.2 & 133,3 & 130,3 & 121,3 \\
$60-64$ & 136,6 & 124,9 & 148,5 & 122,9 \\
$65-69$ & 139,5 & 112,0 & 143,9 & 100,9 \\
$70-74$ & 113,1 & 83,2 & 100,5 & 77,4 \\
$75-79$ & 81,0 & 58,9 & 70,3 & 53,9 \\
$80-84$ & 37,3 & 31,1 & 33,3 & 30,2 \\
$85-89$ & 14,6 & 11,0 & 13,3 & 13,9
\end{tabular}

annähernd gleich werden. Von diesem Zeitpunkte an vermindert sich die Sterblichkeit der Franen, während sie bei den Männern bis zum 69. Jahre ziemlich konstant bleibt. Dann allerdings nimmt sie auch ab, und beide Sterblichkeitsziffern nähern sich während der Periode 75-89. Es erweckt den Anschein, als könnte eine Frau mit grösserer Wahrscheinlichkeit einer Erkrankung an Krebs entgehen, wenn sie das 60. Jahr erreicht, als ein Mann, da dieses Lebensalter bei Männern die höchste Sterblichkeit an Krebs aufweist.

Für den Zählbezirk war das durchschnittliche Todesalter an Krebs für das Jahr 1900 58,1 Jahre. Im Jahre 1890 betrug es 57,2 Jahre. Wenn man nur diejenigen Fälle berücksichtigt, in denen der Tod erst nach dem 15. Lebensjahre eintrat, so betrug die durchschnittliche Lebensdauer für 190058,3 und für 189057,5 .

Wenn man die Lebensalter von 65 Jabren und darüber in Tabelle 7 betrachtet, so kann man sehen, dass die Ziffern dort am höchsten waren, wo die Mütter aus Russland stammten: 654,6; aus Schottland: 533,8; aus Deutschland: 530,4; die Ziffer war dort am niedrigsten, wo die Mütter aus den Vereinigten Staaten stammten: 349,4; aus Ungarn: 280,9; aus Polen: 263,5.

Tabelle 8 zeigt, dass die Sterblicbkeit an Krebs am höchsten bei Personen von 65 Jahren und darüber war; in diesem Alter war sie böber bei Fraven $(487,6)$ als bei Männern $(417,0)$ und höher in den Städten $(494,2)$, als in den ländlichen Bezirken $(412,9)$ der Zählstaaten. 
Tabelle 7 .

Mortalitätsziffer für Krebs auf 100000 der Bevölkerung nach dem entsprechenden Alter und dem Geburtsort der Mutter.

\begin{tabular}{|c|c|c|c|}
\hline \multirow{2}{*}{ Geburtsort d. Mutter } & \multicolumn{3}{|c|}{ Alter } \\
\hline & $15-44$ & $45-64$ & 65 und darüber \\
\hline Schottland & 27,2 & 151,0 & 533,8 \\
\hline Frankreich. & 23,9 & 212,1 & 428,3 \\
\hline Deutschland & 22,8 & 226,2 & 530,4 \\
\hline Irland. & 22,2 & 215,1 & 452,1 \\
\hline Russland & 21,6 & 245,3 & 654,6 \\
\hline England und Wales. & 21.4 & 171,5 & 415,5 \\
\hline Canada... . . & 17,1 & 176,0 & 434,0 \\
\hline Skandinavien & 16,1 & 148,1 & 371,8 \\
\hline Vereinigte Staaten & 14,7 & 145,1 & 349,4 \\
\hline Italien . . . . & 14,5 & 115,0 & - \\
\hline Ungarn und Böhmen & 13,4 & 209,0 & - \\
\hline Polen . . . . & 9,4 & $88, \tilde{2}$ & 263,5 \\
\hline
\end{tabular}

Tabolle 8.

Mortalität an Krebs für den Zählbezirk, mit seinen Unterabteilungen während des Tähljahres 1900 in 5 Gruppen auf 100000 der Bevölkerung, bei entsprechendem Alter und Geschlecht.

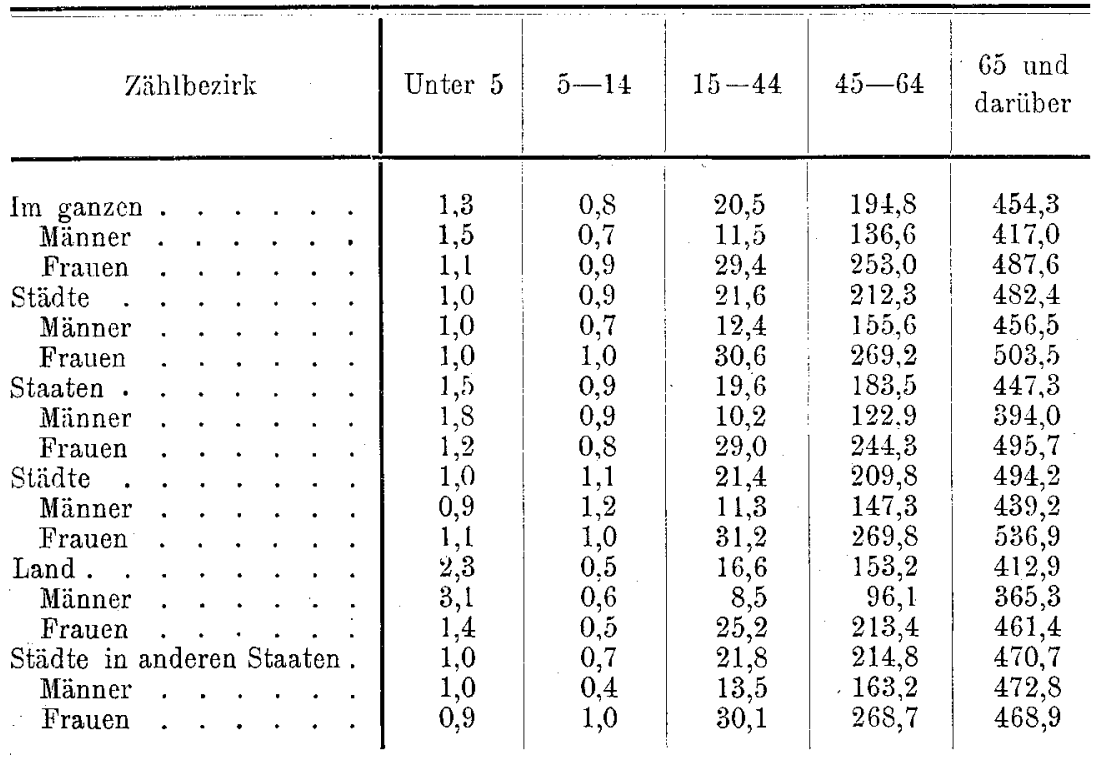

Tabelle 9 zeigt, dass die Mortalität der Verheirateten beiderlei schlechts zwischen dem 15. und 44. Jahre höher war, als das der Unverheirateten. Ueber 44 Jahre war die Mortalität für die Unverheirateten 
Tabelle 9 .

Mortalität der einzelnen Altersgruppen nach Eheverhältnissen geordnet.

\begin{tabular}{|c|c|c|c|c|c|c|c|c|c|}
\hline \multirow[b]{2}{*}{ Geschlecht } & \multicolumn{3}{|c|}{$15-44$} & \multicolumn{3}{|c|}{$45-64$} & \multicolumn{3}{|c|}{65 und darüber } \\
\hline & 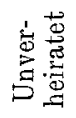 & 今. & 站递 & 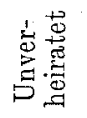 & 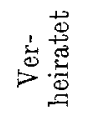 & 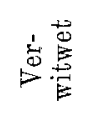 & 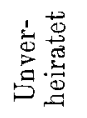 & 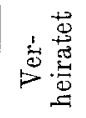 & $\underbrace{\infty}_{0}$ \\
\hline $\begin{array}{l}\text { Im ganzen } \\
\text { Männer. } \\
\text { Frauen. }\end{array}$ & $\begin{array}{r}8.7 \\
6,5 \\
11,3\end{array}$ & $\begin{array}{l}28,6 \\
15,7 \\
39,8\end{array}$ & $\begin{array}{l}69,6 \\
40,3 \\
80,7\end{array}$ & $\begin{array}{l}192,1 \\
142,1 \\
248,8\end{array}$ & $\begin{array}{l}175,6 \\
125,8 \\
238,8\end{array}$ & $\begin{array}{l}256,4 \\
200,8 \\
275,9\end{array}$ & $\begin{array}{l}518,7 \\
512,5 \\
523,1\end{array}$ & $\begin{array}{l}426,0 \\
388,0 \\
496,7\end{array}$ & $\begin{array}{l}455,8 \\
433,7 \\
464,9\end{array}$ \\
\hline
\end{tabular}

beiderlei Geschlechts höher als für die Verheirateten, und bei 65 Jahren und darüber war sie höher bei den Unverheirateten beiderlei Geschlechts, als bei den Verheirateten oder Verwitweten.

\section{Der Krebs verschiedener Organe.}

Tabelle 10 zeigt für das Zählgebiet die Mortalitätsziffer der einzelnen Altersstufen für den Krebs bestimmter Organe oder Organgruppen auf 1000 Todesfälle in demselben Alter und nach dem Geschlecht geordnet.

Ta $b$ e 11 e 10.

Verhältnis der Todesfälle an Krebs für bestimmte Organe.

\begin{tabular}{|c|c|c|c|c|c|c|c|c|c|c|c|}
\hline \multirow[b]{2}{*}{ Organ oder Körperteil } & \multicolumn{3}{|c|}{ Alle Alter } & \multicolumn{3}{|c|}{ 24-44 Jahre } & \multicolumn{3}{|c|}{$45-64$ Jahre } & \multicolumn{2}{|c|}{65 J. u. } \\
\hline & 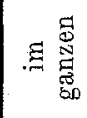 & 芦 & 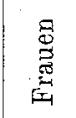 & 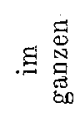 & $\underset{8}{9}$ & 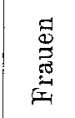 & 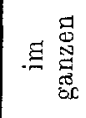 & 岂 & 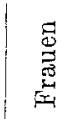 & . & 离 \\
\hline dor & 82,8 & 92,4 & 76,9 & 78,6 & 18,9 & 62,6 & 79,2 & 92,2 & 71,5 & 38,4 & 83. \\
\hline ase . & 12,5 & 25,3 & 6 & 5,8 & 6,3 & 5 & 12.3 & 22,8 & 6 , & 19,3 & 34 \\
\hline hirn & 2,0 & 2,6 & 1 & 4,4 & 4,7 & 4 & 1,1 & 1,6 & 0,7 & 1,4 & 9 \\
\hline irust. & 一 & 7,5 & 157,8 & $=$ & 11,0 & 151,9 & - & 7,8 & 157,8 & - & 6 \\
\hline uge. & 1,3 & 2,4 & 0,7 & - & $\rightarrow$ & $\ldots$ & 0,3 & 0,4 & 0,2 & 2,5 & 4 \\
\hline ienitalien & - & 9,6 & 6,3 & - & 3,1 & 8,1 & - & 8,6 & 5,8 & $\ldots$ & 12. \\
\hline iopf, Gesicht u. Nacken & 59,2 & 104,2 & 31,4 & 37,3 & 92,3 & 15,5 & 46,2 & 95,1 & 17,4 & 87,9 & 118 \\
\hline r & 4,9 & 1 & 1 & 4 & 7,8 & 2 & 5,4 & 13,1 & 1 & 4,8 & 9 \\
\hline eber & 133,4 & 145,6 & 125,9 & 112,3 & 159,6 & 93,6 & 133,7 & 151,4 & 123,3 & 143,2 & 132, \\
\hline Intere Extremitäten & 2,7 & 3,7 & 2,1 & 2,7 & 4,7 & 1 , & 2,0 & 2,4 & 1,7 & 3,6 & 4, \\
\hline ungen & 5,5 & 6,1 & 5 & 4,9 & 6,3 & 4, & 7,4 & 8,6 & 6,7 & 2,9 & 3. \\
\hline Iund, Zunge u. Hals & 46,8 & 95,5 & 16,8 & 25,3 & 67,3 & 8, & 45,4 & 98,3 & 14,2 & 59,6 & 101, \\
\hline Ivarien . . . . & - & - & 9,7 & - & 一 & 16,7 & 一 & 一 & 8,9 & - & - \\
\hline 'enis . & - & 3,9 & - & - & 4,7 & 一 & - & 3,7 & 一 & - & 3 \\
\hline ectum & 42,9 & 54,9 & 35,5 & 54,2 & 79,8 & 44,0 & 39,8 & 50,6 & 33,5 & 42,6 & 53, \\
\hline lagen. & 315,6 & 430,6 & 244,7 & 249,6 & 427,2 & 179,2 & 318,8 & 441,4 & 246,4 & 348,0 & 422 \\
\hline 'estikel. . . & - & 1,6 & - & - & 4,7 & - & - & 0,8 & - & - & 1 \\
\hline bere Extremitäten & 1,9 & 3,3 & 1,1 & 1,3 & 1,6 & 1,2 & 0,9 & 1,2 & 0,7 & 3,9 & 6 ; \\
\hline Iterus & - & - & 276,8 & - & 一 & 399,9 & 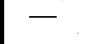 & $一$ & 304,2 & 一 & 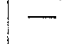 \\
\hline
\end{tabular}


Diese Tabelle zeigt auch, dass in je 1000 Todesfällen von Männern in allen Altersstufen mit bestimmt lokalisiertem Krebs

430,6 verursacht waren durch Krebs des Magens,

\begin{tabular}{|c|c|c|c|c|c|}
\hline 145,6 & $n$ & $n$ & $n$ & $n$ & der Leber, \\
\hline 104,2 & $n$ & $n$ & $"$ & $n$ & des Kopfes, Gosichts und Nackens, \\
\hline 95,5 & $n$ & $n$ & $y$ & $n$ & des Mundes, der Zunge und des Halses, \\
\hline 92,4 & $"$ & $n$ & $n$ & $n$ & des Unterleibs, \\
\hline 54,9 & $n$ & " & $n$ & $n$ & des Mastdarms. \\
\hline
\end{tabular}

Unter Frauen verhielt sich der Krebs auf 1000 Todesfälle derart, dass 276,2 verursacht waren durch Krebs des Uterus,

\begin{tabular}{|c|c|c|c|c|c|}
\hline 244,7 & $n$ & $"$ & $"$ & $\eta$ & des Magens, \\
\hline 157,8 & $n$ & $n$ & $n$ & $m$ & der Brust, \\
\hline 125,9 & $"$ & $n$ & $"$ & $"$ & der Leber, \\
\hline 76,9 & $"$ & $n$ & $"$ & $"$ & des Unterleibs, \\
\hline 35,5 & $n$ & $\eta$ & $n$ & $\eta$ & des Mastdarms. \\
\hline
\end{tabular}

Das Ueberwiegen der Todesfälle an Krebs bei den weiblichen Genitalund Brustorganen reduziert das Verhältnis bei anderen Organen oder Teilen im Vergleich mit den entsprechenden Verhältnissen bei Männern. Wenn man aber den Krebs jener Organe unberücksichtigt lässt, so übertrifft die Mortalität an anderen Formen des Krebses bei Männern diejenige bei Frauen.

Tabelle 11 teilt die 17296 Todesfälle an Krebs im Zählbezirk nach den Organen oder Körperteilen, sowie nach Geschlecht und Farbe ein.

Krebs der Brust. Die Mortalität auf 100000 der Bevölkerung war viel höher bei den Frauen $(9,1)$, als bei den Männern $(0,3)$. Sie war auch viel höher für die Altersgruppe zwischen 45 und 64 Jahren (30,7), als in niederem Alter, und noch höher bei 65 Jahren und darüber (Franen 59,6; Männer 3,0).

Die Mortalitätsziffer ans dieser Ursache war im ganzen genommen höher bei weissen Frauen $(9,1)$ als bei farbigen $(7,9)$. Unter 45 Jahren war sie bei farbigen Frauen $(7,2)$ höher als bei weissen $(3,9)$, aber nach diesem Alter war sie wieder bei weissen Frauen höher. Bei weissen Franen zwischen 45 und 64 Jahren verhielt sich die Mortalität ungefähr gleich bei Eingeborenen $(30,3)$, wie bei denen fremder Herkunft $(30,9)$. Von 65 Jahren an war das Verhältnis höher für Eingeborene $(66,6)$; für die aus dem Auslande stammenden $(50,1)$.

Krebs der Leber. Die Angaben zeigen, dass die Mortalität für alle Altersstufen höher war bei Frauen $(7,2)$ als bei Männern $(5,2)$, und dieser Unterschied war besonders ausgesprochen in den Altersstufen zwischen 45 und 64 Jahren (Frauen 24,0; Männer 17,1). Da das primäre Lebercarcinom entschieden ungewöhnlich ist, entsprechen die oben angegebenen 
Tabelle 11 .

\begin{tabular}{|c|c|c|c|c|c|c|c|c|c|c|}
\hline & & & & \multirow{2}{*}{ 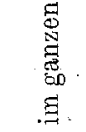 } & \multirow{2}{*}{ 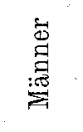 } & \multirow{2}{*}{ 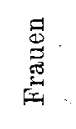 } & \multicolumn{2}{|c|}{ Weisse } & \multicolumn{2}{|c|}{ Farbige } \\
\hline & & & & & & & M. & $\mathrm{H}^{\mathrm{t}}$ & M. & $\mathrm{F}$ \\
\hline Abdomen. & & • & & 1107 & 471 & 636 & 463 & 631 & 8 & 5 \\
\hline Blase. & . & . & & 180 & 129 & 51 & 125 & 49 & 4 & 2 \\
\hline Gehirn . & . & . & . & 27 & 13 & 14 & 12 & 14 & 1 & 二 \\
\hline Brust... & . & . & . & 1344 & 38 & 1306 & 36 & 1256 & 2 & 50 \\
\hline Extremitäten & . & . & $\cdot$ & 62 & 36 & 26 & 33 & 25. & 3 & 1 \\
\hline Auge.. . & . & . . & - & 18 & 12 & 6 & 12 & 6 & - & - \\
\hline Genitalien & &. & . . & 101 & 49 & 52 & 48 & 51 & 1 & 1 \\
\hline Kopf, Gesicht & $t$ und & Nack & & 791 & 531 & 260 & 521 & 255 & 10 & 5 \\
\hline Nieren. . & . & . & 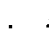 & 90 & 41 & 49 & 40 & 47 & 1 & 2 \\
\hline Larynx & 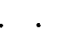 & . & . & 66 & $5 \check{5}$ & 11 & 55 & 11 & - & 一 \\
\hline Leber. & & 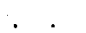 & 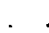 & $178 t$ & 742 & 1042 & 720 & 1024 & 22 & 18 \\
\hline Lungen & . . & . & . & 73 & 31 & 42 & 30 & 40 & 1 & 2 \\
\hline Mund, Zunge & und & Hals &. & 626 & 487 & 139 & 477 & 133 & 10 & 6 \\
\hline Ovarien . & . . & . . & - & 80 & - & 80 & - & 76 & 一 & 4 \\
\hline Penis '. & .. & . . & . & 20 & 20 & - & 20 & - & - & - \\
\hline Rectum & 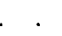 & . & . & 574 & 280 & 294 & 271 & 279 & 9 & 15 \\
\hline Magen. & 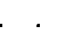 & - & . & 4220 & 2195 & 2025 & 2140 & 1964 & 55 & 61 \\
\hline Testikel & . & . . & & 8 & 8 & - & 7 & - & 1 & - \\
\hline Uterus . . & 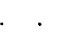 &.$\quad$. & . & 2291 & - & 2291 & 一 & 2164 & - & 127 \\
\hline Unbestimmt . & . . & . & . & 3834 & 1250 & 2584 & 1223 & 2500 & 27 & 84 \\
\hline
\end{tabular}

Ziffern dem, was wir hier erwarten würden, insofern nämlich die grösste Zahl der Krebsfälle bei Frauen zwischen 45 und 64 Jahren vorkommt. Da wir ja wissen, dass Frauen in diesem Alter wehr zum Carcinom neigen als Männer, so dürfen wir bei ihnen von vornherein eine grössere Häufigkeit von Metastasen in der Leber erwarten.

Krebs des Kopfes, des Gesichtes und des Nackens. Von der Gesamtsumme der Fälle in dieser Abteilung fielen 531 auf. Männer und 260 auf Frauen. Die Mortalität für diese Gruppe war viel höher bei Männern $(3,7)$, als bei Frauen $(1,8)$ und war am höchsten bei Personen von 65 Jahren und darüber (Männer 38,6; Frauen 23,5).

Krebs des Mundes, der Zunge und des Halses. Dieser kam bei llännern $31 / 2$ mal so oft vor, als bei Frauen. . Von 626 Fällen ereigneten sich $57 \mathrm{im}$ Alter. zwischen 20 und 44 Jahren, 300 zwischen dem 45. und 60. Jahr, $263 \mathrm{im}$.65. Jahr und darüber. Die Mortalität war für Männer wie Frauen am höchsten im Alter. von 65 Jahren aufwärts (Männer 33,1; Frauen 9,7). Diese Resultate in Verbindung mit denen aus der unmittelbar vorhergehenden Gruppe können als eine weitere Stütze für die Theorie angesehen werden, die eine chronische Reizung als wichtigen ätiologischen Faktor annimmt, besonders mit Bezug auf den Ursprung der oberflächlichen Carcinome. Die natürliche Schlussfolgerung wäre, dass die grösste Zahl der obigen Fäle vom Typus des Plattenepithelioms sein 
müsste. Diese Abart zeigt in so vielen Fällen eine chronische Reizung in der Vorgeschichte, dass es scheint, als müsste man eine solche Aetiologie ernsthaft in Betracht ziehen.

Krebs des Magens. Grösser als bei irgend welcher anderen Ursache war die Zahl der Todesfälle an Krebs in dieser Gruppe. Von der Gesamtsumme der Todesfälle an Krebs, nämlich 17296 , waren 4220 die Folge von Magenkrebs $(24,4 \mathrm{pCt}$ ). Beide Geschlechter waren annähernd gleichmässig betroffen (2195 Männer und 2025 Frauen). Die Mortalität war sehr niedrig bei Personen unter 45 Jahren und war mehr als zweimal so hoch bei Personen von 65 Jahren als bei solchen zwischen 45 und 64 Jabren. Bei Personen von 65 Jahren und darüber war die Mortalität höher bei Männern $(138,3)$ als bei Frauen $(105,9)$; viel höher bei Weissen $(122,4)$ als bei Farbigen $(105,9)$ und weiterhin auch höher unter den aus dem Auslande stammenden Weissen $(153,2)$, als bei den eingeborenen Weissen $(96,6)$.

Krebs des Uterus. Unter allen Todesfälen von Krebs handelte es sich in 2291 um Krebs des Uterus. Das bedeutet einen Prozentsatz von 7,9 auf sämtliche Fälle berechnet und einen Prozentsatz von 12,6, wenn wir nur die Krebstodesfalle bei Frauen in Betracht ziehen. Bei Frauen von 20 bis 44 Jahren betrug die Mortalität 10,7 pCt. Am höehsten war sie bei Frauen im Alter von 45 bis 64 Jahren $(59,1)$, blieb aber noch hoch bei 65 Jahren und darüber. Sie war viel höher bei den Farbigen, als bei den Weissen in allen Altersgruppen, und höher bei den eingeborenen Weissen zwischen 45 und 64 Jahren $(59,6)$, als bei den eingewanderten $(55,5)$.

\section{Die Beziehungen des Krebses zur Beschäftigung.}

Die folgende Liste zeigt für die Zählstaaten die Mortalität an Krebs bei Männern und Frauen auf 100000 der Bevölkerung nach Berufsarten geordnet.

Durchschnittszahl für alle beschäftigten Männer .

Akademisch Gebildete.

Geistliche . . . . . . . . . . 102,2

Juristen . . . . . . . . . . . . . 52,5

Aerzte . . . . . . . . . . . . . . 67,5

Bureau-Angestellte . . . . . . . . . . . . . . 37,3

Buchhalter und Korrespondenten . . . . . 28,0

Bankbeamte und Spekulanten . . . . . . 41,4

Kassierer, Auktionatoren und Agenten . . . 58,1

Kaufmanns- und Handelsstand . . . . . . . 52,0

Kaufleute und Händler . . . . . . . . 81,3

Trödler und Hansierer . . . . . . . . 62,7 
Hotelwirtschaft

Hotel- und Pensions-Besitzer . . . . . . 60,1

Café- und Restaurant-Besitzer. . . . . . 28,0

Oeffentlicher Dienst, Polizei und Militär . . . 442,9

Pförtner und Küster.. . . . . . . . . 66,7

Schutzmämer, Nachtwächter und Detektives . 60,3

Arbeitende und dienende Klasse . . . . . . 66,5

Arbeiter (ausser Landarbeitern) . . . . . 126,6

Diener . . . . . . . . . . . . . . 44,3

Manufaktur und mechanische Industrie . .. . . 53,3

Bäcker und Konditoren ... . . . . . . 51,0

Schmiede . . . . . . . . . . . . . 107,3

Tapezierer . . . . . . . . . . . 72,6

Eisen- und Stahl-Arbeiter . . . . . . . 31,5

Maschinisten . . . . . . . . . . . . 52,2

Maurer und Steinmetze . . . . . . . . 98,0

Weber. . . . . . . . . . . 34,5

Maler . . . . . . . . . . . . . . 45,0

Schneider. . . . . . . . . . . . . 58,4

Landwirtschaft, Transport und andere Berufe im

Freien . . . . . . . . . . . . . . 69,2

Kärrner, Drescher, Fuhrleute usw. . . . . 22,6

Bauern, Pflanzer und Landarbeiter . . . . 87,7

Gärtner und Blumenzüchter . . . . . . 72,9

Stallknechte . . . . . . . . . . . . 46,1

Berglente und Steinhauer . . . . . . . 38,4

Matrosen und Fischer usw. . . . . . . . 113,1

Durchschnit für beschäftigte Franen. . . . . . . . . . 49,7

Lehrerimen . . . . . . . . . . . : 47,8

Buchhalterinnen und Comptoiristimnen . . . 15,1

Wäscherimen . . . . . . . . . . 25,3

Krankenwärterinuen und Hebeammen . . . 105,0

Dienerinnen . . . . . . . . . . . . 99,6

Arbeiterinnen der Textilbranche . . . . . 9,9

Schneiderinnen . . . . . . . . . . 88,4

In Tabelle 12 geben wir den Prozentsatz der Mortalität an Krebs im Verhältnis zur Gesamtzabl aller Todesfälle wieder und zwar 1 . in den Vereinigten Staaten, 2. im Zählbezirk mit seinen Unterabteilungen. 
Tabelle 12.

\begin{tabular}{|c|c|c|c|}
\hline & $\begin{array}{c}\text { Weisse } \\
\text { Männer } \\
\text { pCt. }\end{array}$ & $\begin{array}{c}\text { Farbige } \\
\text { Männer } \\
\text { pCt. }\end{array}$ & $\begin{array}{c}\text { Frauen } \\
\text { pCt. }\end{array}$ \\
\hline 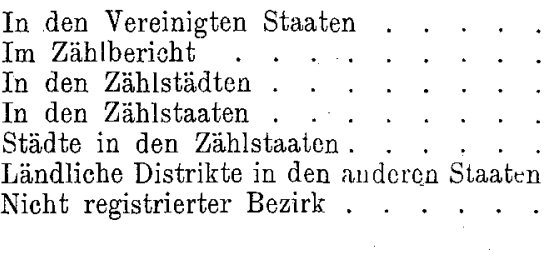 & $\begin{array}{l}3,63 \\
3,86 \\
3,71 \\
3,80 \\
3,50 \\
4,20 \\
3,30 \\
3,30\end{array}$ & $\begin{array}{l}0,78 \\
1,30 \\
1,34 \\
1,36 \\
1,54 \\
0,65 \\
1,29 \\
0,60\end{array}$ & $\begin{array}{l}4,40 \\
5,64 \\
5,37 \\
5,97 \\
5,86 \\
6,61 \\
5,11 \\
3,22\end{array}$ \\
\hline
\end{tabular}

\section{Die geographische Verbreitung des Krebses in den Vereinigten Staaten.}

Tabelle 13 teilt das Territorium der Vereinigten Staaten in die einzelnen grossen geographischen Gruppen auf. Innerhalb dieser Gruppen erscheint der Prozentsatz der Krebsmortalität im Massstab zn je 1000 Todesfüllen geordnet. Ferner werden die Prozentzahlen auch für beide Geschlechter, sowie für Stadt- und Landbevölkerung gegeben.

Tabelle 13 .

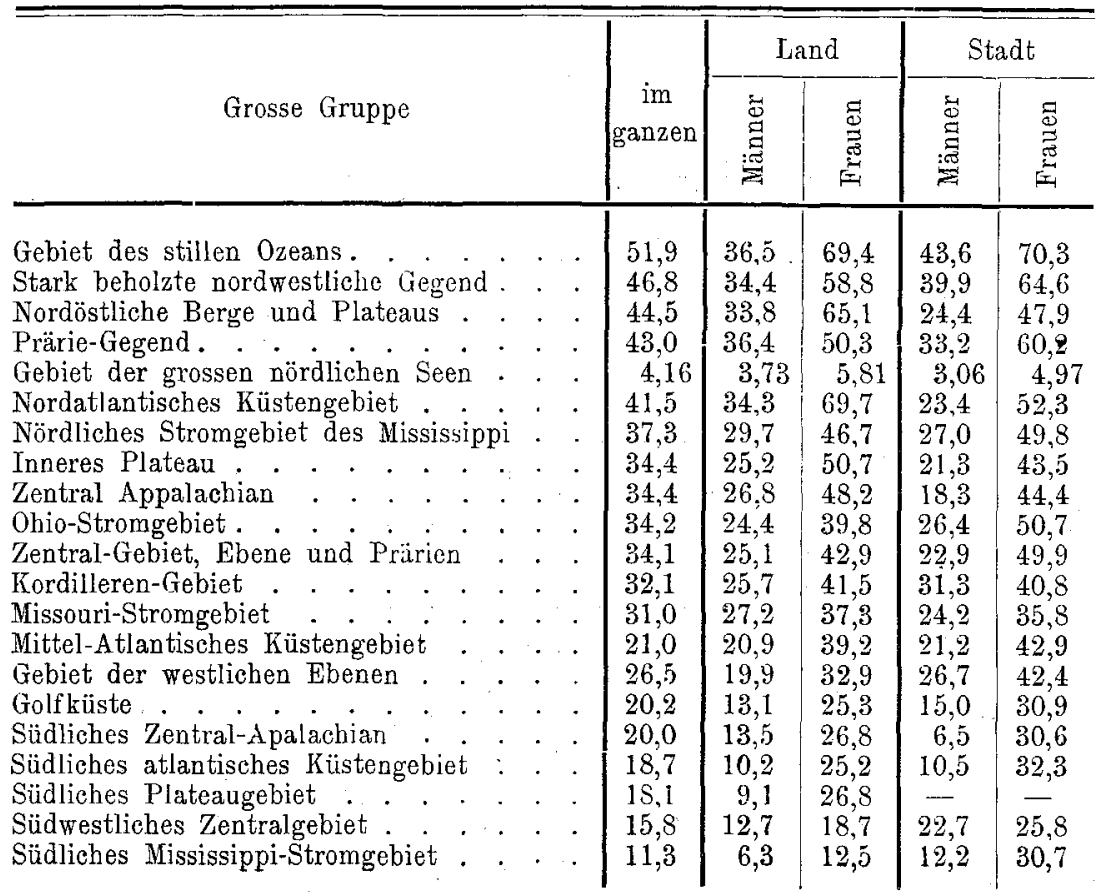


Nach dieser Tabelle war das Verhältnis der Todesfälle an Krebs am grössten im Küstengebiet des Stillen Ozeans (51,9), in den stark beholzten Gegenden des Nordwestens (46,8), auf den nordöstlicben Bergen und Plateaus $(44,5)$, irn Prairiegebiet $(43,0)$. Am niedrigsten war es im inneren Plateau des Südens $(18,1)$, im Zentralgebiet des Südwestens $(15,8)$ und im Nordmississippigürtel $(11,3)$.

Bei der Besprechung einiger Statistiken der Vereinigten Staaten sagt ein Autor (British Medical Journal, 2. Mai 1903): "Man wird finden, dass die Nationalitätenfrage eine Erklärung abgibt für die sehr hohe Sterblichkeit in denjenigen Staaten, in denen die grössere Anzahl von Emigranten aus Europa sich niederlässt." Sie beweist auch nach Ansicht dieses Verfassers, dass ,augenscheinlich die Atlantischen Staaten, die alljährlich eine grosse Menge von Auswanderern aus England und Deutschland aufnehmen, aus diesem Grunde allein eine durchaus höhere Krebsfrequenz aufweisen müssen, als die von einer eingeborenen Bevölkerung bewohnten Staaten." Indessen werden diese Folgerungen nicht durch die Tatsachen gestützt. Vergleichen wir gewisse grosse Gruppen mit Bezug auf die Zahl der Eingeborenen, so finden wir die in Tabelle 14 gelieferten Verhältnisse.

Tabelle 14.

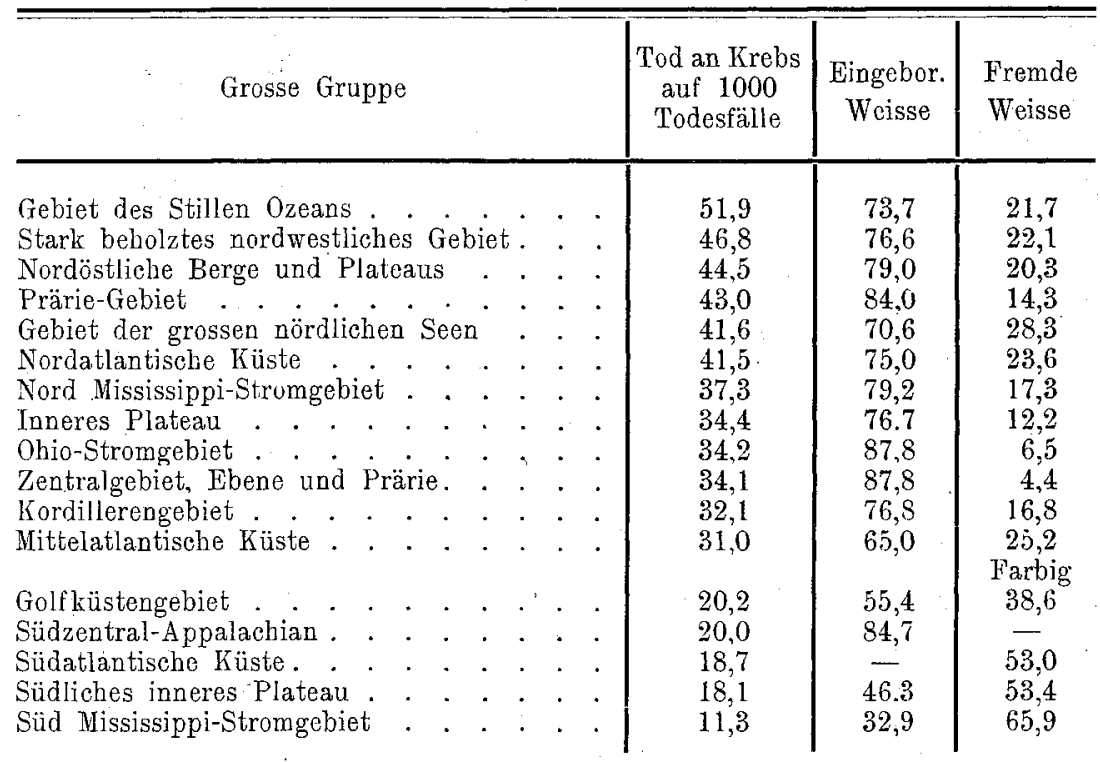

Aus dieser Tabelle ist ersichtlich, dass die zwei Gebiete, die den grössten Prozentsatz fremder Bevölkerung aufweisen, hinsichtlich der Krebssterblichkeit die fünfte bzw. zwölfte Stelle einnehmen. Ferner ist das 
südliche Carolina-Appalachian-Gebiet das viertniedrigste und seine Bevölkerung ist zusammengesetzt aus $84,7 \mathrm{pCt}$. eingeborener Weissen; dagegen beläuft sich die Bevölkerung an eingeborenen Weissen im Prairiegebiet auf $84 \mathrm{pCt}$, während es die vierthöchste Stelle einnimmt. In den südlichen Staaten, wo die farbige Bevölkerung sehr gross ist, ist die durchschnittliche Krebsfrequenz am niedrigsten. Dies ist auch zu erwarten, wenn man die geringere Sterblichkeit an Krebs in dieser Rasse kennt. In einer kürzlich erschienenen Arbeit zieht $\mathrm{Hyde}$ den Schluss, dass "physiologische Pigmentierung der Haut eine relative Immunitat dieses Organs gegen Krebs zn bedingen scheine;" und auch dass "die farbigen Rassen augenscheinlich weniger am Krebs anderer Organe, als der Haut, zu leiden haben, als die Weissen. Diese relative Immunität mag bedingt sein durch den Schntz, den das Pigment des Integuments gewährt gegen die aktinischen Strahlen des Liehts." Aus Tabelle 14 scheint also hervorzugehen, dass die Mortalität an Krebs in den verschiedenen Teilen der Vereinigten Staaten keine direkte Beziehung habe zur Zahl der fremden Weissen innerhalb der Bevölkerung.

Bei der Betrachtung einer Krebskarte der Vereinigten Staaten wird unsere Aufmerksamkeit sofort dureh die Tatsache erregt, dass mit der einzigen Ausnahme von Californien das Gebiet hinsichtlich der Mortalität an Krebs unter je 1000 Fällen, in denen die Todesursache gegeben ist, in zwei grosse Abteilungen getrennt werden kann. Südlich vom 37. Breitengrade beläuft sich die Krebssterblichkeit auf weniger als 16 pro 1000. Darin sind inbegriffen Arizona, New Mexico, Texas, Indian Territory, Arkansas, Mississippi, Louisiana, South Carolina, Georgia und Florida, der westliche Teil von Tennessee und der nördliche von Alabama. In den folgenden Gebieten ist die Frequenz ein.wenig höher, vom 16. bis zum 24. Breitengrade, nämlich die Küstenlinie von Texas; Louisiana und Mississippi, der südliche Teil von Alabama und das frühere Territorium Oklahoma.

Nördlich vom 37. Breitengrade in jenem Teile zwischen dem MissouriFluss und.Californien sowie der westlichen Balfte der Staaten Wasbington und Oregon schwankt die Freqnenz zwischen 25 und 39 pro 1000 Trodesfalle. Oestlich des Missouri bis zur Atlantischen Küste beträgt die Mortalität $31-39$, whihrend sie in einigen Staten dieser grossen Region auf 40 und darüber steigt.

\section{Zusammenfassiuig.}

Im Zählgebiet stand im Jahre 1890 Krehs an dreizehnter Stelle auf der Liste der Todesursachen und die Rate betrug 47,9 auf 100000 der Bevölkerung. Im Jahre 1900 rückte der Krebs bis zur siebenten Stelle binauf mit einer Rate von 60 . 


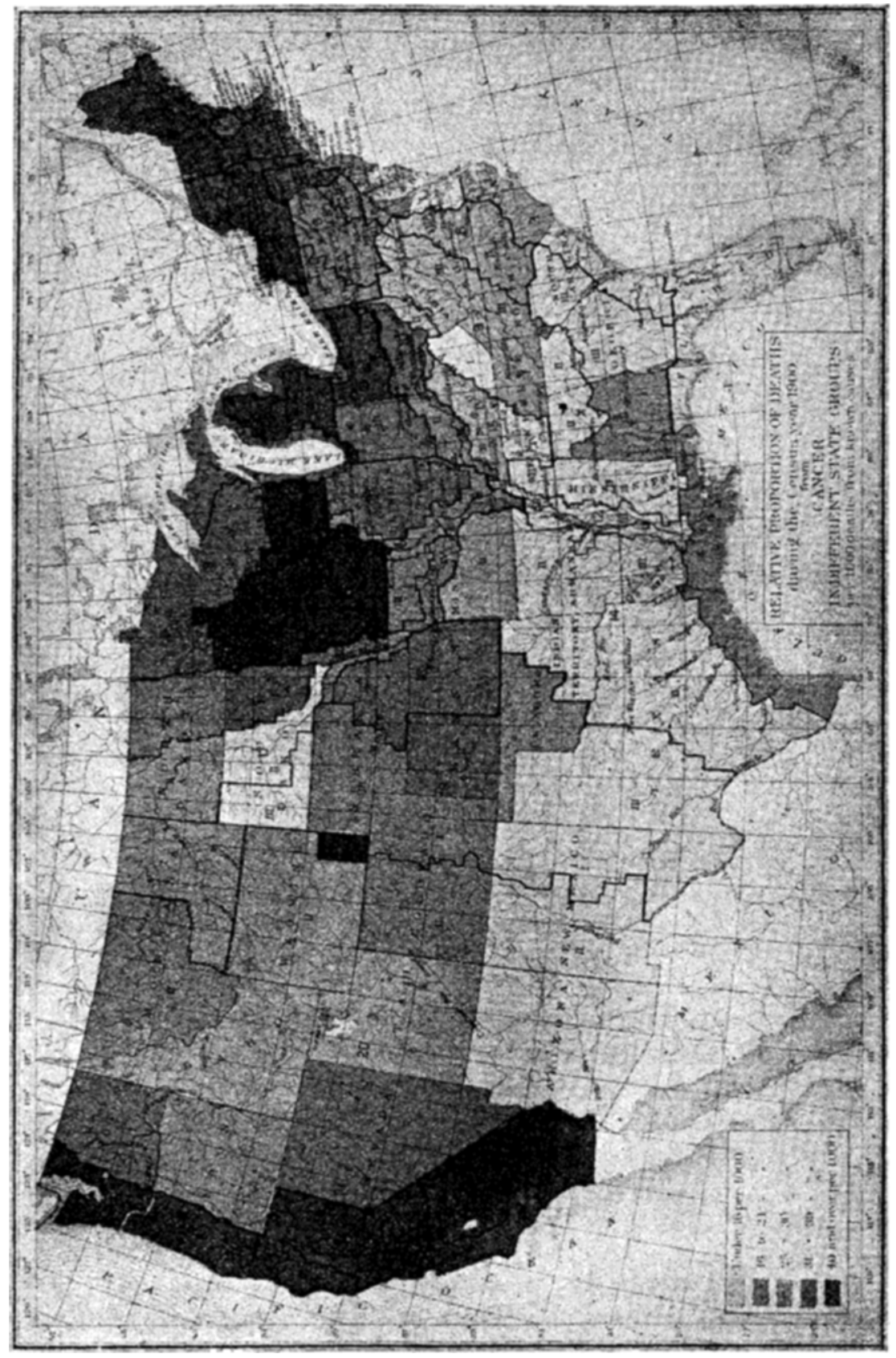


In den Vereinigten Staaten war im Jahre 1900 das Verhältnis der Todesfälle an Krebs 29,5 unter je 1000 Fällen, in denen die Todesursache bekannt war. Im Jahre 1890 betrug das entsprechende Verhältnis 22,5. In England und Wales betrug 1899 die Frequenz 82,9. Dies bedeutete eine Zunahme von 15,3 auf 100000 in den Jahren 1890--99, während im Zählgebiet der Vereinigten Staaten von 1890-1900 eine nur wenig kleinere Vermehrung von 12,1 zu konstatieren war.

Tabelle 15 .

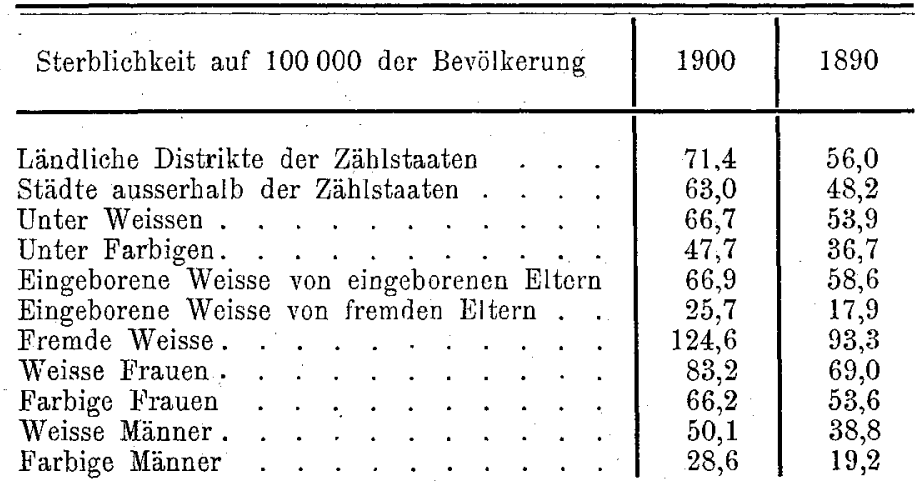

T a b e lle 16.

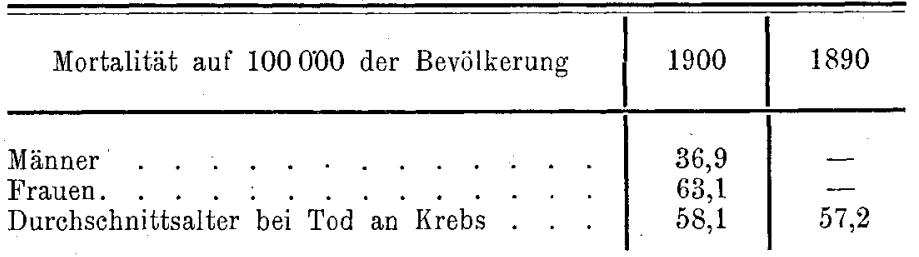

Grösste Sterblichkeit mit 65 Jahren und zwar bei Frauen höher als bei Männern.

Die Mortalität war am höchsten bei denen, deren Mütter in Russland, Schottland oder Deutschland geboren waren, an niedrigsten bei denen, die in den Vereinigten Staaten, Ungarn oder Polen geboren waren. Etwaige Einflüsse von seiten der Väter konnten nicht in Betracht gezogen werden, da genane Nachweise aus begreiflichen Gründen vielfach nicht zu erheben waren.

Wenn wir den Einfluss von Lebensalter und Khestand auf die Krebsmortalität betrachten, so scheineu unsere Statistiken zu ergeben, dass beide Geschlechter etwa gleichmässig beeinflusst werden. Während nämlich die Verbeirateten im Alter von 15-44 Jabren häufiger der Krankheit zum Opfer fallen, als die Ledigen gleichen Alters, kehrt sich vom 45. Jahre an 
das Verhältnis um; von jetzt an sind es die Ledigen, die einen grösseren Prozentsatz von Krebstodesfällen aufweisen, als die Verheirateten.

In je 1000 Todesfällen von Männern aller Altersstufen an bestimmt lokalisiertem Krebs waren

430,6 bedingt durch Krebs des Magens,

\begin{tabular}{|c|c|c|c|c|}
\hline 145,6 & " & $n$ & $"$ & der Leber; \\
\hline 104,2 & $n$ & $n$ & $n$ & des Kopfes, Gesichtes und Nackens, \\
\hline 95,5 & $n$ & $n$ & $n$ & des Mundes, der Zunge und des Halses, \\
\hline 92,4 & 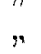 & $n$ & $n$ & des Abdomens, \\
\hline 54,9 & $"$ & $n$ & $"$ & des Rektums; \\
\hline
\end{tabular}

bei Frauen

276,2 bedingt durch Krebs des Uterus,

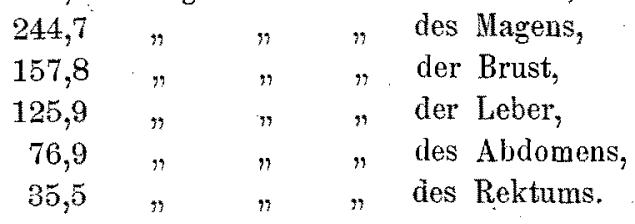

Bei der Berücksichtigung der Berufsarten findet man grosse Schwankungen in der Krebssterblichkeit. Mit einer einzigen, später zn erwähnenden Ausnahme ist die Sterblichkeit an Krebs am höchsten unter denen, die schwere Arbeit im Freien verrichten. An der Spitze stehen die nicht landwirtschaftlichen Tagelöhner (Strassenarbeiter, Eisenbahnarbeiter usw.) mit einer Frequenz von 126,6 auf 100000 der Bevölkerung, dann Matrosen und Fischer mit 113,1, Hufschmiede mit 107,3, Maurer mit 98 und Landarbeiter mit 87,0. Die einzige Ausnahme findet sich bei den Geistlichen mit der überraschend hohen Prozentziffer von 102,2. Man könnte diese Tatsache damit erklären, dass diese Klasse gewöhnlich ein höheres Alter erreicht, als die Augehörigen der meisten anderen Berufszweige. Im übrigen aber scheint eine direkte Bezielung zwischen Beschäftigung und Krebssterblichkeit zu bestehen. Schwierig ist es zu sagen, was diese Erscheinung mit schwerer Arbeit und dem Aufenthalt im Freien za tun bat, wenn man sie nicht auf die Theorie der chronischen Reizung beziehen will. Bevor man dies beweisen könnte, müsste man freilich erst die Lokalisierung der Neubildung in jedem einzelnen Falle kennen.

Gegen die Theorie, dass der Alkohol die Entstehung des Krebses fördere, lässt sich einwenden, dass die Durchschnittziffer für diejenigen Berufszweige, die in irgend welcher Beziehung zu offentlichen Lustbarkeiten stehen, nur 35,3 beträgt. Dies ist die niedrigste aller Gruppen. Die Klasse der Café- und Restaurantbesitzer hat sogar nur einen Durchschnitt von 28,0 . Hätte der andauernde Genuss von Bier und anderen alkoholischen 
Getränken irgend etwas mit der Krankheit zu tư, so müsste ein höherer Mittelwert gefunden werden.

Dass der Biergenuss keinen direkten Finfluss auf die Krebsfrequenz hat, kann man auch dadureh zeigen, dass man die Werte für gewisse Städte, die überwiegend ron Deutschen bewohnt werden und die die Zentren des Brauereigewerbes darstellen, mit einander vergleicht. In Milwaukee betrug die Frequenz anf 1000 Todesfälle 52,5, in Cincimati 44,5 , in St. Louis 40,3 . Und doch rangieren diese Städte hinter San Franciseo mit 58,7, Syracuse mit 57,2, Sprinfield mit 56,2, Hartford mit 53,9 u. a. und stehen ungefähr gleich mit Detroit mit 49,4 , Los Angeles mit 47,6, Buffalo mit 47,0 und New Haven mit 46,8 .

Die Ansicht vieler Forseher, dass der Krebs häufiger den Flussläufen folgt, wird durch unsere Tabellen nicht unterstützt. Diejenigen Gebiete in den Vereinigten Staaten, die an den grossen Strömen liegen, z. B. dem Mississippi, Missouri, Ohio, Rio Grande u. a., haben, mit wenigen Ausnahmen, eine niedrigere Sterblichkeit, als die anderen Teile des Landes. Dagegen sind die einzigen gemeinsamen Punkte für die weit zerstreuten Gebiete mit hoher und höchster Sterblichkeit die, dass diese Gegenden fast ausnahmlos hügelig oder gebirgig sind; weiterhin aber sind sie noch stark beholzt und durch zahlreiche kleine Flussläufe oder Teiche gut bewässert.

Fin Vergleich unserer Statistiken mit denen europäischer Länder ergibt, dass die Einwanderer in grösserer Zahl an Krebs zugrunde gehen, als ihre Landslente, die daheim geblieben sind. Es muss aber hervorgehoben wnrden, dass diese erhöhte Sterblichkeit doch wohl nur eine scheinbare ist. Die überwiegende Mehrzahl der Einwanderer sind nämlich bereits erwachsen; wenn sie unser Land betreten; sie befinden sich demnach bereits innerhalb des „krebsfähigen" Alters und müssen einen höheren Prozentsatz an Krebstodesfällen ergeben, wenn man ihren Schicksalen statistisch nachforseht. In den betreffenden Heimatsländern jenseits des Ozeaus wird dagegen eine jede statistisehe Erhebung auf die Gesamtbevölkerung ausgedehnt, welehe auch alle Kinder umfasst, die das Krebsalter noch nicht erreicht haben. Dort wird demgemäss der Prozentsatz niedriger ausfallen müssen. Der einzige Weg, auf dem diese Werte vergliehen werden könnten, wäre der, die Mortalitätsziffer einer grossen Zabl von Emigranten mit einer entsprechenden Zahl gleichen Alters in ihrem eigenen Lande zu vergleichen.

\section{Schlussfolgerungen.}

1. Die Krebskrankheit scheint in den letzten zehn Jahren in den Vereinigten Staaten eine Zunahme in der Mortalität von 12,1 auf 100000 der Berölkerung erfahren zu haben. 
2. Die Mortalität ist in den ländlichen Distrikten und kleinen Städten böher, als in den grossen Städten.

3. Eingeborene Weisse werden viel weniger ergriffen, als die fremden Weissen.

4. Einwanderer weisen eine höhere Mortalitätsrate an Krebs auf, als ihre Landsleute daheim; indessen ist diese Differenz nur eine scheinbare. 5. 63,1 pCt. aller Krebsfälle entfallen auf Frauen und 36,9 pCt. auf Yänner.

6. Diejenigen, welche schwere Arbeit im Freien verrichten, zeigen eine grössere Sterblichkeit an Krebs, als diejenigen mit siizender Beschäftigung.

7. Die Gebiete mit der grössten Sterblichkeitsziffer sind die gebirgigen, wohl beholzten und bewässerten Gegenden.

8. Entlang den grossen Flussläufen findet sich anscheinend kein Ueberwiegen der Krankheit.

9. Der Genuss von Alkohol scheint keinen Einfluss auf die Krebskrankheit zu haben; wenigstens zeigen Städte mit starker deutscher Bevölkerung und grossen Brauereibetrieben keine entsprechende Vermehrung der Krebssterblicbkeit.

10. Die Krebssterblichkeit ist am höchsten bei Personen von 65 Jahren and darüber.

11. Das durchschnittliche Todesalter beträgt 58,1 Jahre. 